\title{
Anti-matrix metalloproteinase-9 DNAzyme decreases tumor growth in the MMTV-PyMT mouse model of breast cancer
}

\author{
Miranda A Hallett ${ }^{1}$, Bin Teng ${ }^{1}$, Hisashi Hasegawa', Luciana P Schwab², Tiffany N Seagroves ${ }^{2}$ and \\ Tayebeh Pourmotabbed ${ }^{1 *}$
}

\begin{abstract}
Introduction: Despite continued improvements in diagnosis, surgical techniques, and chemotherapy, breast cancer patients are still overcome by cancer metastasis. Tumor cell proliferation, invasion and metastasis are mediated, at least in part, through degradation of basement membrane by neutral matrix metalloproteinases (MMP) produced by tumor and stromal cells. Evidence suggests that MMP-9 plays a significant role in breast tumor cell invasion and metastasis. DNAzymes or catalytic oligonucleotides are new classes of gene targeting molecules that bind and cleave a specific $m R N A$, resulting in decreased protein expression.

Methods: The application of anti-MMP-9 DNAzyme (AM9D) for the treatment of primary and metastatic breast cancer was evaluated in vitro and in vivo using MDA-MB-231 cells and the MMTV-PyMT transgenic breast cancer mouse model. Spontaneously developed mammary tumors in MMTV-PyMT transgenic mice were treated intratumorally with naked AM9D, once a week for 4 weeks. The stability of DNAzyme was determined in vitro and in vivo using fluorescently labeled DNAzyme.

Results: AM9D specifically inhibited expression of MMP-9 in MDA-MB-231 cells resulting in reduced invasive property of these cells by $43 \%$. Weekly intratumoral treatment of spontaneously developed mammary tumors in MMTV-PyMT transgenic mice was sufficient to significantly reduce the rate of tumor growth and final tumor load in a dose dependent and statistically significant manner $(P<0.05)$. This decrease in tumor growth was correlated with decreased MMP-9 protein production within the treated tumor tissues. Tumors treated with AM9D were also less vascularized and contained more apoptotic cells compared to control and untreated tumors.
\end{abstract}

Conclusions: These results show that targeting and down regulation of MMP-9 by AM9D could prove useful as a therapy against breast carcinoma tumor growth and invasion.

\section{Introduction}

Breast cancer is one of the leading causes of cancer death in women, second only to lung cancer [1-3]. The majority of morbidity and mortality amongst cancer patients is due to metastasis of tumor cells to distant organs [2,4]. Breast cancer most commonly metastasizes to bone, lymph nodes, lung, liver, and brain [5]. Despite continued improvements in diagnosis, surgical techniques, and chemotherapy, lethality from breast cancer remains high.

\footnotetext{
* Correspondence: tpourmotabbe@uthsc.edu

'Department of Microbiology, Immunology and Biochemistry, University of Tennessee Health Science Center, 858 Madison Ave., Memphis, TN 38163, USA

Full list of author information is available at the end of the article
}

Matrix metalloproteinase-9 (MMP-9) production by tumor and stromal cells is one of the most important factors for metastatic behavior of tumor cells [6-8]. MMP-9 is a member of the metzincin family of enzymes, which play an important role in normal physiological responses, including wound healing and bone formation [9]. MMP-9 becomes deregulated during tumorigenesis and is associated with pro-oncogenic events such as neo-angiogenesis, tumor cell proliferation and metastasis [10]. High level of MMP-9 expression in breast cancer is positively correlated with enhanced tumor cell invasion and metastasis [11,12] and with enhanced progression and poorer prognosis [10].
C Biomed Central

(c) 2013 Hallett et al.; licensee BioMed Central Ltd. This is an open access article distributed under the terms of the Creative Commons Attribution License (http://creativecommons.org/licenses/by/2.0), which permits unrestricted use, distribution, and reproduction in any medium, provided the original work is properly cited. 
MMP-9 is conserved across several species (human, chimpanzee, dog, cow, mouse, rat, chicken, zebrafish, and Arabidopsis thaliana). MMP-9 degrades type IV collagen, one of the most abundant collagens in the extracellular matrix (ECM) [13], which may stimulate local invasion, the first step in metastasis. In addition, MMP-9 also cleaves pro-cytokines, chemokines, and growth factors, thereby modifying their biological activity [14-16]. The downregulation of MMP-9 has been shown to increase $\beta 1$-integrin expression, leading to activation of extracellular signal-regulated kinases (ERKs) and increasing apoptosis through one of two mechanisms: (1) release of cytochrome $C$ into the cytosol and/or (2) increase in nuclear factor $-\kappa \mathrm{B}(\mathrm{NF}-\kappa \mathrm{B})$ activation, followed by activation of caspase-3 [17]. Although few normal cell types express MMP-9 under normal physiological conditions, the majority of human metastatic tumor cells that have been tested consistently show elevated MMP-9 activity compared with benign control cells, including melanoma, fibrosarcoma, breast adenocarcinoma, and glioma [18-21]. In addition, tumor cells that stably express MMP-9 cDNA have been shown to have enhanced metastastic ability [22]. Thus, inhibition of MMP-9 expression could be a useful therapeutic modality to decrease the growth and invasive properties of tumor cells.

RNA-cleaving phosphodiester-linked DNA based enzymes (DNAzymes) are catalytic DNA molecules that specifically bind to and cleave targeted mRNA in a sequence-specific manner. The result is efficient degradation of the mRNA transcript, and thus, similar decreased expression levels of the encoded protein $[23,24]$. Catalytic oligonucleotides have emerged as novel, highly selective inhibitors or modulators of gene expression [25]. Khachigian and colleagues have reported that the DNAzymes targeting early growth response factor-1 (Egr1) mRNA inhibit neointimal formation after balloon injury to the rat carotid artery wall and reduce intimal thickening after stenting of pig coronary arteries [26]. DNAzyme targeting c-Jun causes repair of injured carotid arteries in rats [27]. Finally, a DNAzyme targeting vascular endothelial growth factor receptor 2 (VEGFR2) significantly inhibits the growth of breast tumors derived from xenografting of MDA-MB435 cells into nude mice by inducing apoptosis [28].

Here, we examine the effects of a novel anti-MMP9 DNAzyme (AM9D) on breast tumor growth in the mouse mammary tumor virus-driven polyoma virus middle $\mathrm{T}$ oncoprotein transgenic (MMTV-PyMT) mouse model of breast cancer. We demonstrate for the first time that once-weekly intratumoral injection of AM9D in the absence of any carrier molecule, for four weeks, was sufficient to significantly reduce the rate of tumor growth and final tumor load in a dose-dependent and statistically significant manner $(P \leq 0.05)$. Together, the data presented here justify the further development of AM9D for its potential as an anti-tumor agent and as an ideal candidate for breast cancer therapy.

\section{Materials and methods DNAzyme}

All DNA oligonucleotides used in these experiments were synthesised by Integrated DNA Technology (Coralville, IA, USA). DNAzymes were designed according to the specific rule of 10-23 DNAzyme [29]. The DNAzyme targeting MMP9 mRNA contains a catalytic domain of 15 highly conserved deoxynucleotides flanked by two substrate-recognition domains. The sequence of the DNAzyme targeting mRNA of mouse and human MMP-9 is 5'-GTGGTGCCAGGCTAGC TACAACGATTGAGGTCG-3'. In the control DNAzyme, 5'CTAGTCAGCGGCTAGCTACAACGATAAGCTGCT 3', the catalytic sequence of DNAzyme is flanked by nine bases randomly chosen and not specific for any MMP coding sequence. In some cases, the DNAzyme was end-labeled with Alexa Fluora C5-melamide 633 or Oregon Green ${ }^{\mathrm{TM}} 488$ C5-maleimide (Invitrogen, Carlsbad, CA, USA) using T4 Polynucleotide kinase, as suggested by the manufacturer's protocol.

\section{Cell transfection}

MDA-MB-231 human breast tumor cell lines (ATCC, Manassas, VA, USA) were plated in DMEM supplemented with $10 \%$ fetal bovine serum (FBS) and allowed to grow to 80 to $90 \%$ confluence at $37^{\circ} \mathrm{C}$ with $5 \% \mathrm{CO}_{2}$. The cells were then serum-starved for 4 hours prior to transient transfection with Oregon Green ${ }^{\mathrm{TM}} 488$-maleimidelabeled AM9D or control DNAzyme $(24 \mu \mathrm{g})$ using Lipofectamine 2000 (Invitrogen). After 18 hours incubation at $37^{\circ} \mathrm{C}$ in serum-free medium, cells were collected and sorted, and the transfected cells were isolated for further analysis.

\section{Analysis of MMP9, MMP1, MMP13, MMP14, MMP19 and MMP21 mRNA levels in transfected cells}

The MMP9, MMP1, MMP13, MMP14, MMP19 and MMP21 mRNA expression levels in the DNAzymetransfected cells were quantified by reverse transcription-polymerase chain reaction (RT-PCR) using specific MMP9 (forward primer; 5-GCAGGAATGCGGCTCT GG-3', reverse primer; 5'-CCCGTCGAAGGGATACC3'), MMP1 (forward primer; 5'-CATTCTACTGATATCGG-3', reverse primer; 5'-AGAAAACAGAAATGAAA-3'), MMP13 (forward primer; 5-GAC TTCCCA GGAATTGGTGA-3, reverse primer; 5-TGA CGCGAACAATACGGTTA-3'), MMP14 (forward primer; 5'GAGCTCAGGGCAGTGGATAG -3', reverse prime; 5'CCACCTCAATGATGATCACC -3'), MMP19 (forward 
primer; 5'-GGGTCCTGTTCTTCCTACAT-3', reverse primer; 5 CAATCCTGCAGTACTGGTCT-3'), and MMP21 (forward primer; 5'-AACAATAGGACACGCTATGG-3', reverse primer; 5'-CATCTCTTTTCCATGTCCAG-3') primers [30]. Total RNA from the transfected cells was isolated by Trizol reagent (Invitrogen) and reverse-transcribed with random hexamer primers (Promega, Madison, WI, USA) using MMLV-RT enzyme (Invitrogen, Carlsbad, CA).

Mouse or human $B A C T$ ( $\beta$-actin) mRNA was also amplified as internal controls, with corresponding (human forward; 5'-CAAGAGATGGCCACGGCGGCT3', human reverse; 5'-TCCTTCTGCATCCTGTCAGCA3', mouse forward; 5'-CAGGAGATGGCCACTGCCG CA-3', mouse reverse; 5'-AAGCACTTGCGGTGCACGATG-3') primers. The PCR products were subjected to $2 \%$ agarose gel and visualized by ethidium bromide staining. Expression was quantified by an Alpha Imager 2000 documentation and analysis system (Alpha Innotech Corporation, San Leandro, CA, USA).

\section{Analysis of MMP-9 activity by gelatin gel zymography}

MDA-MB-231 cells were transiently transfected with AM9D or control DNAzyme in serum-free medium as stated above. Twenty-four hours post transfection-media were collected and concentrated 10-fold using Amicon Ultracell filtration units (Millipore, Co Cork, Ireland). Protein concentration of the collected media was determined by Bradford dye binding techniques (a standard Bio Rad assay) using bovine serum albumin as a standard. The MMP-9 activity in the culture media was then assessed by gelatin zymography [31].

\section{Cell invasion assay}

Cells were transfected with fluorescently labeled AM9D or control DNAzyme for 18 hours in serum-free media as above. The fluorescent positive cells were identified by flow cytometry, isolated and seeded in ECMatrix ${ }^{\mathrm{TM}_{\mathrm{M}} \text { invasion }}$ chambers (Millipore, Billerica, MA, USA). After 24 hours incubation at $37^{\circ} \mathrm{C}$ with $5 \% \mathrm{CO}_{2}$, the number of cells that migrated through the ECM layer and attached to the polycarbonate membrane was quantified spectrophotometerically at $560 \mathrm{~nm}$ according to the manufacturer's protocol. The assays were done in multiples and the differences in the values between groups were evaluated by analysis of variance (ANOVA). $P<0.05$ was considered significant.

\section{In vitro stability of DNAzyme}

AM9D was incubated in $\mathrm{PBS}$ at $37^{\circ} \mathrm{C}$, and an equal amount was removed at various time points and incubated with $M M P 9$ mRNA at $37^{\circ} \mathrm{C}$. After a 2 -hour incubation the RNA samples were visualized on a $4 \%$ urea-polyacrylamide gel. For DNAzyme cellular uptake and stability, MDAMB-231 cells were cultured on cover-glass slides. Cells were then transfected with $4 \mu \mathrm{g}$ fluorescently labeled DNAzyme, as described above, fixed with formaldehyde at 24,48 , or 72 hours post transfection and visualized by confocal microscopy. The nucleus was visualized by 4',6diamidino-2-phenylindole (DAPI)/anti-fade.

\section{Animals}

All animal experiments were conducted following approval by the University of Tennessee Health Science Center Institutional Animal Care and Use Committee (IACUC). Friend virus B-type (FVB)/Nj female mice were obtained from Jackson Laboratory (Bar Harbor, ME, USA) and crossed with PyMT-positive FVB males. The offspring were genotyped by real-time PCR on a Roche LC 480 LightCycler using the following primers and universal probe library (UPL) probe \#11 (forward primer: 5' AACCCGAGTTCTCCAACAG 3; reverse primer: 5' TCAGCAAC ACAAGGATTTC 3') to identify MMTVPyMT-positive females. Female mice were palpated once a week beginning at approximately 4 weeks of age and palpable tumors were measured in two dimensions (longest diameter and shortest width) with digital calipers. Tumor volume was calculated using the formula:

\section{Tumor volume $=$ Width $^{2} \times$ Length $/ 2$}

When each transgenic female developed at least three palpable tumors of dimensions of $3 \mathrm{~mm} \times 5 \mathrm{~mm}$, which typically occurred at 8 weeks of age, each tumor was injected intratumorally with either 10 or $25 \mu \mathrm{g}$ of AM9D or control DNAzyme suspended in PBS in a total volume of $5 \mu \mathrm{l}$, using a Hamilton syringe mounted with a PT2, 26G needle. Tumors identified at week 0 were injected once per week for a total of 4 weeks of therapy, and the site of intratumoral injection was varied to ensure that all areas of the tumor were exposed to the AMD9 or control DNAzyme. Palpable mammary tumors that arose after week 1 in other mammary glands of the same mice were left untreated. For each cohort, transgenic females with a combined number of at least nine tumors of comparable size were utilized (AMD9, $25 \mu \mathrm{g}, n=2$ mice and 12 mammary tumors and control DNAzyme, $n=3$ mice and 9 mammary tumors). An independent cohort of animals was also included in tumor endpoint volume studies, in which additional mice were treated with either control DNAzyme (25 $\mathrm{g}$, $n=3$ mice and 15 mammary tumors) or AM9D (AM9D, $10 \mu \mathrm{g}, \mathrm{n}=2$ mice and 9 mammary tumors; $25 \mu \mathrm{g}, n=2$ mice and 9 mammary tumors).

Tumor growth was monitored weekly by caliper measurement. All animals were euthanized one week after the last DNAzyme treatment (typically at 12 weeks of age). At necropsy, tumors were removed, final tumor dimensions were measured by calipers and the tumor 
wet weight was determined. Tumors were then either flash frozen in liquid nitrogen, or fixed in $4 \%$ paraformaldehyde overnight, followed by cryoprotection in $25 \%$ sucrose for several days. Cryoprotected tumors were then washed with $0.1 \%$ PBS prior to embedding in optimal cutting temperature (OCT) compound and preparation of 8-micron sections.

For analysis of Mmp9 mRNA expression levels in tumors, OCT compound-embedded tumor sections were scraped from glass slides of individual control DNAzyme or AM9D-treated tumors to form a pool of tumor material, and total RNA and cDNA was prepared and analyzed by RT-PCR analysis as described above.

\section{Immunohistochemistry}

Mammary tumor vasculature was visualized using rat anti-mouse CD31 antibody (1:50) (BD Biosciences, San Jose, CA, USA) and Alexa Fluor-594 goat anti-rat IgG (H $+\mathrm{L})$ secondary antibody (Invitrogen). Stromal cells (myofibroblasts) were detected using anti- $\alpha$-smooth muscle actin ( $\alpha$-SMA) antibody at 1:250 dilution (Sigma, St. Louis, MO) and Alexa Fluor 488 goat anti-mouse IgG2a (Invitrogen) secondary antibody at 1:500 dilution. MMP9 protein was detected using a rabbit anti-mouse MMP-9 antibody at a 1:200 dilution (Santa Cruz Biotechnology, Santa Cruz, CA) followed by Alexa Fluor-594 goat antirabbit IgG antibody (1:500). Digital images were captured using a Bio-Rad Confocal Laser Scanning Microscope, using the Lasersharp 2000 software. Image J imaging analysis software was used for measurement of MMP-9,
CD31-immunostained endothelial area (EA), and caspase-3-positive cells in the scanned immunohistochemistry (IHC) sections of mammary tumors. According to Chantrain et al. [32], compared with the so-called hot spot and the random fields methods, the EA measurement method is more reproducible for quantification of tumor vasculature.

\section{Statistical analysis}

All data are expressed as mean \pm SD or standard error (SE). Data were analyzed with SSPS software (SigmaStat version 2.03) using one-way analysis of variance (ANOVA), or Student's $t$-test. Tumor growth over time among three groups was analyzed by two-way ANOVA using Prism software (Graphpad version 4.0b, La Jolla, $\mathrm{CA})$. In all cases, $P$-values $<0.05$ were considered statistically significant.

\section{Results}

AM9D treatment specifically reduces MMP-9 production and suppresses the invasive behavior of breast tumor cells in vitro

The specificity of AM9D toward MMP9 mRNA was demonstrated in MDA-MB-231 human breast cancer cells. MDA-MB-231 cells express MMP1, MMP9, MMP13, MMP14, MMP19, and MMP21 (Figure 1A, lane 3). As shown in Figure $1 \mathrm{~A}$ and $1 \mathrm{~B}$, contrary to control DNAzyme (lane 2), AM9D treatment (lane 1) significantly decreased the activity (Figure $1 \mathrm{~B}$ ) and the level of MMP9 mRNA (Figure 1A) in MDA-MB-231 cells
A

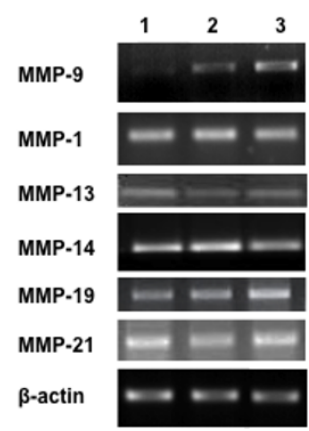

B

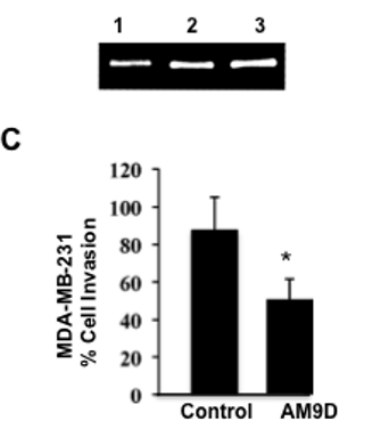

Figure 1 Effect of AM9D treatment on metalloproteinase (MMP) expression in MDA-MB-231 cells. (A) Expression levels of MMP9, MMP1, MMP13, MMP14, MMP19, and MMP21, and BACT (B-actin) mRNA in MDA-MB-231-transfected cells. MDA-MB-231 cells were transfected with Oregon Green 488-labeled DNAzymes, control DNAzyme or mock transfection reagents as described in Materials and methods. Positively transfected cells were identified by flow cytometry. Total RNA was isolated and MMP9, MMP1, MMP13, MMP14, MMP19 and MMP21, and BACT (Bactin) mRNA were amplified by reverse-transcription (RT)-PCR and the PCR products were subjected to agarose gel and visualized by ethidium bromide staining. Lane 1, AM9D; lane 2, control DNAzyme; lane 3, cells treated with DOTAP (N-[1-(2,3-Dioleoyloxy)]-N,N,N-trimethylammonium propane methylsulfate) transfection reagent only. (B) Gelatin zymography of culture media from transfected MDA-MB-231 cells. The cultured media from MDA-MB-231 cells transfected with AM9D (lane 1), control DNAzyme (lane 2), or treated with DOTAP alone (lane 3) were separated on $8 \%$ SDS polyacrylamide gel containing $1 \mathrm{mg} / \mathrm{ml}$ gelatin. (C) Histogram showing the percentage of carcinoma cells invading the ECMatrix ${ }^{\mathrm{TM}}$ matrigel matrix after treatment with AM9D compared to cells treated with control DNAzyme. Cells were transfected with Oregon Green 488 labeled-DNAzymes, sorted and cultured in a matrigel matrix invasion chamber as described in Materials and methods. ${ }^{*} P<0.05$ compared with control (one-way analysis of variance). 
without having an effect on $M M P 1, M M P 13, M M P 14$, MMP19 or MMP21 mRNA levels. Although MMP-2 and -3 have also been reported to contribute to breast tumorigenesis [30], we did not detect $M M P 2$ or $M M P 3$ mRNA expression in cultured MDA-MB-231 cells. These data demonstrate that the AM9D therapy is specific as it only affects the production of MMP-9 in cells, and that reduction of $M M P 9$ mRNA leads to reduction in enzymatic activity, as expected.

The effect of decreased MMP9 mRNA expression on the invasive behavior of MDA-MB-231 cells was assessed by transfecting the cells with fluorescently labeled AM9D or control DNAzyme and determining the invasive behavior of the sorted cells using the ECMatrix ${ }^{\mathrm{TM}}$ invasion chamber. As shown in Figure 1C, the mean invasion potential of MDA-MB-231 decreased by approximately $43 \%$ when transfected with AM9D compared to control DNAzyme-treated cells. These data are consistent with the reports of others demonstrating that MMP-9 is one of the key mediators of tumor cell invasion $[11,12,33]$ and supports the idea of the DNAzyme gene-targeted approach for MMP-9 as a breast cancer therapeutic agent.

MMP-9 is expressed in mammary tumors and the associated stroma in the MMTV-PyMT model

The MMTV-PyMT transgenic mouse model is a widely used pre-clinical model of estrogen and progesterone receptor-negative luminal-like breast cancer with welldefined stages of progression and metastasis to lung $[34,35]$. More importantly, mammary adenocarcinomas exhibit changes in biomarkers similar to those observed in patients with breast cancer $[34,36]$. On a pure FVB/ $\mathrm{Nj}$ strain background, all PyMT-positive females will eventually develop mammary tumors in each of their ten mammary glands, although the time of tumor onset varies among individual glands [35]. The expression patterns of various MMPs in the PyMT model [37-39] are also similar to those observed in patients diagnosed with ductal mammary adenocarcinoma [40]. Therefore, this model was chosen to ascertain the role of AM9D as a pharmacologic inhibitor of MMP-9.

To confirm the presence of MMP-9 protein in latestage mammary carcinomas, tumors were harvested from MMTV-PyMT transgenic females at 12 weeks of age. Tumor sections were stained with antibodies to both $\alpha$-SMA, a marker for stromal myofibroblasts, and MMP-9. IHC analysis demonstrated the presence of MMP-9 in the tumor epithelium, including areas highly populated with stromal fibroblasts (Figure 2). It is also likely that MMP-9 is produced by the tumor-associated macrophages that are known to be present in PyMT tumors $[41,42]$.

\section{DNAzyme is stable in vitro and in vivo and is present in mammary tumors for at least 14 days post single intratumoral injection}

Prior to testing AM9D for its effect on mammary tumor growth, the in vivo stability and cellular uptake of naked DNAzyme molecules was examined by intratumorally injecting tumor-bearing MMTV-PyMT transgenic female mice with fluorescently labeled AM9D in PBS. The animals were then sacrificed at 7, 10, and 14 days (Figure 3A, a-c) post AM9D injection, and mammary tumors were harvested, sectioned, and viewed under a fluorescent microscope. As shown in Figure 3A, fluorescently-labeled oligonucleotides could be easily detected in a diffuse pattern within the tumor for up to 14 days (Figure 3A, c). Moreover, AM9D could also be detected in adjacent, non-injected mammary tumors of the same
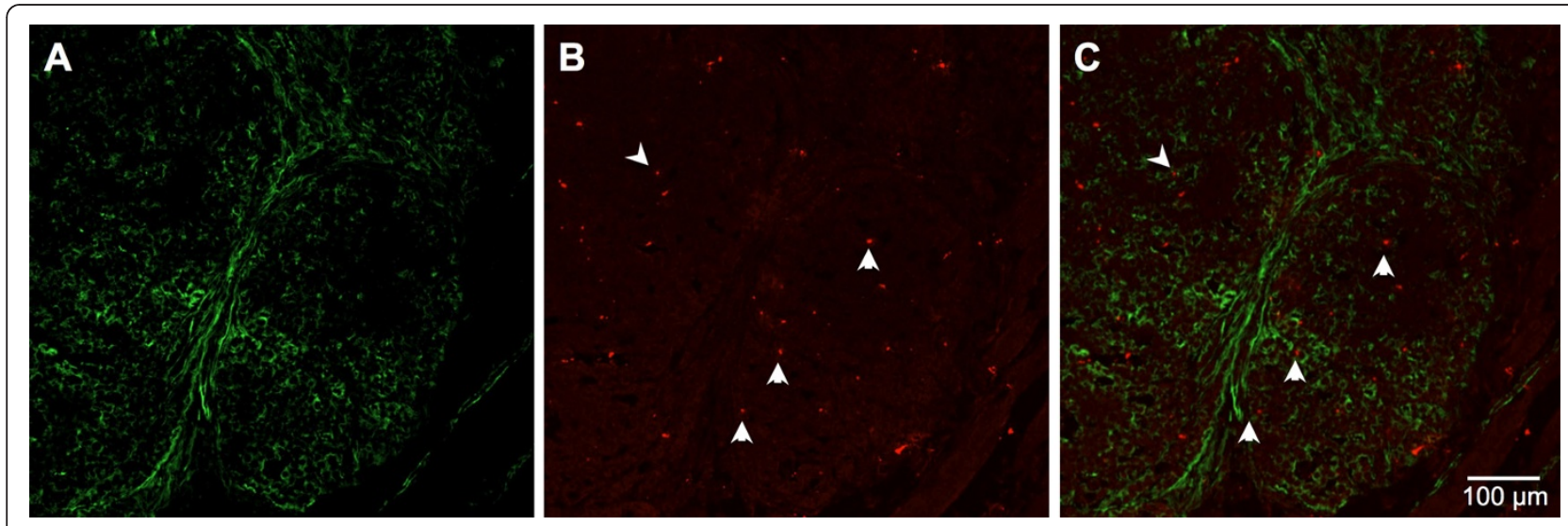

Figure 2 Immunohistochemical staining for metalloproteinase (MMP)- 9 and $\alpha$-smooth muscle actin ( $\alpha$-SMA) in mammary tumor sections. Tumors were resected from mice and double stained with antibodies to $\alpha$-SMA to detect stromal cells (A) and MMP-9 (B). When channels were merged $\mathbf{( C )}$, these data show that MMP-9 was present in both stromal and tumor cells. Magnification 200x; scale bar is equivalent to $100 \mu \mathrm{m}$. 


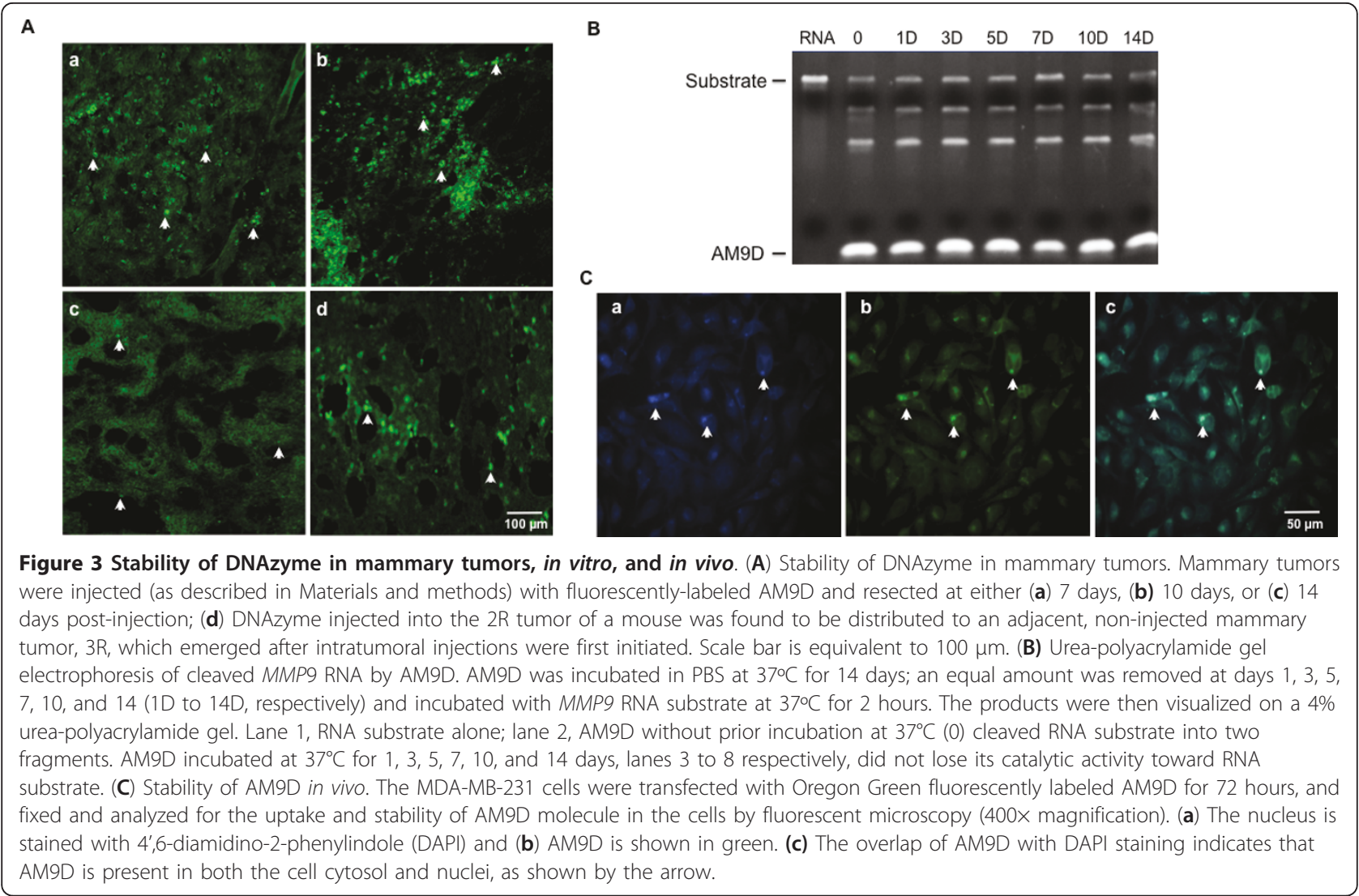

mouse (Figure 3A, d), indicating a wider distribution pattern than might be expected from intratumoral injection. Therefore, the DNAzymes are stable in vivo and can efficiently distribute within the injected tumor and to an adjacent non-injected tumor.

To further examine the stability of the DNAzyme in solution and in vitro, DNAzyme prepared in PBS was incubated for up to 14 days at $37^{\circ} \mathrm{C}$. Aliquots were removed at different time intervals and the amount and activity of DNAzyme remaining over time was determined by applying the DNAzyme to a $6 \%$ urea-polyacrylamide gel and measuring its ability to cleave a $760 \mathrm{bp}$ MMP9 RNA substrate (Figure 3B). As demonstrated in Figure 3B, DNAzyme oligonucleotides are stable in PBS at $37^{\circ} \mathrm{C}$ and no significant degradation or loss of enzymatic activity was observed over the 14 day period.

The in vitro stability of AM9D was further confirmed by transfecting MDA-MB-231 cells grown on slides with fluorescently labeled AM9D as described above, and visualizing the presence of AM9D in cells by fluorescent microscope at 24, 48 and 72 hours post transfection. As shown in Figure 3C, DNAzyme molecules are present in cells for at least 72 hours post transfection and are located in both the cytosol and the nucleus. The nucleus localization significantly increases the effectiveness of
DNAzyme therapy. These data in corroboration with the in vivo stability of AM9D administered to mammary tumors of the MMTV-PyMT transgenic mouse (Figure $3 \mathrm{~A}, \mathrm{c}-\mathrm{d}$ ) demonstrate the retention and potential efficacy of this therapy.

\section{AM9D treatment reduces final tumor load in the MMTV- PyMT tumor model}

The efficacy of AM9D to reduce breast tumor volume in MMTV-PyMT transgenic mice was tested by directly injecting two concentrations (10 or $25 \mu \mathrm{g}$ ) of AM9D or control DNAzyme into mammary tumors of transgenic females bearing at least three tumors per mouse, each at an early palpable size (approximately $3 \mathrm{~mm} \times 5 \mathrm{~mm}$ ), once a week for 4 weeks. Tumor palpations were performed weekly to determine changes in tumor volume over time. The growth rate of AM9D-treated tumors $(n=12)$ was slower than both control DNAzyme-treated tumors $(n=9)$ and untreated tumors $(n=7)$ (Figure 4A). This resulted in a significant reduction in the final tumor volume of AM9D-treated compared to control DNAzyme-treated $(P<0.001)$ and untreated $(P<0.01)$ tumors at age 12 weeks (Figure 4A). In fact, administration of AM9D at $10 \mu \mathrm{g}$ was sufficient to reduce the size of the tumor by $39.5 \%(n=9, P \leq 0.01)$ compared to control 
A

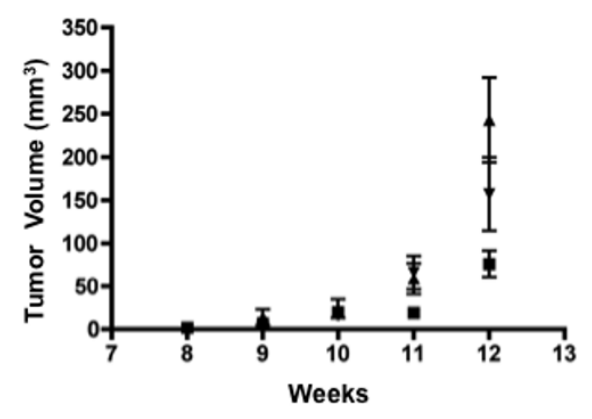

C

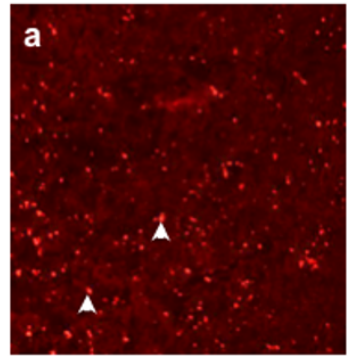

b

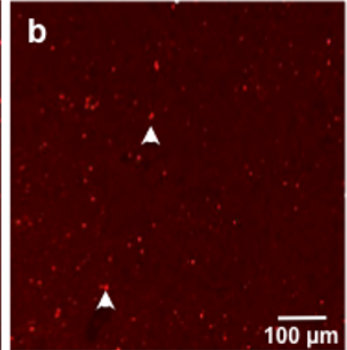

B

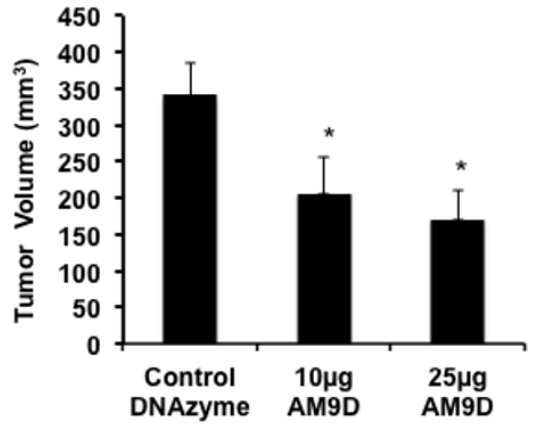

D

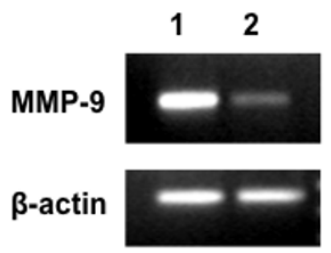

Figure 4 Effect of AM9D on the rate of tumor growth, final mean tumor volume, and metalloproteinase (MMP)-9 expression. (A) AM9D-treated tumors $(\mathbf{-})$ grew at a slower rate than either untreated tumors (no injections) ( $\mathbf{v})$, or tumors treated with control DNAzyme $(\mathbf{\Delta})$; at the study endpoint, age 12 weeks, $P<0.05$. (B) Weekly intratumoral treatment of transgenic mice with $10 \mu \mathrm{g}(n=9$ tumors) or $25 \mu \mathrm{g} \mathrm{AM9D}$ ( $n=$ 21 tumors) per tumor reduced mean tumor burden by $39.5 \%$ or $50.1 \%$, respectively, when compared to tumors treated with control DNAzyme ( $n=24$ tumors) $(P<0.01$ ANOVA). (C) Mammary tumors treated with either $25 \mu \mathrm{g}$ AM9D or control DNAzyme were stained with an MMP-9 antibody. (a) Mammary tumors treated with $25 \mu \mathrm{g}$ control DNAzyme for 4 weeks showed increased MMP-9 staining (arrows) compared to (b) mammary tumors treated with AM9D for 4 weeks. Images are shown at 200x magnification; scale bar is equivalent to 100 um. (D) Mmp9 mRNA expression levels in control DNAzyme- or AM9D-treated tumors. Total RNA was isolated and pooled from optical cutting temperature (OCT) compound-embedded tumor sections scraped from glass slides of individual control DNAzyme- or AM9D-treated tumors. Mmp9 and Bact (Bactin) mRNA were amplified by RT-PCR and the PCR products were subjected to agarose gel and visualized by ethidium bromide staining. Lane 1, AM9D; lane 2, control DNAzyme.

$(n=24)$, which increased to $50 \%$ when $25 \mu \mathrm{g}$ of AM9D was utilized ( $n=21, P \leq 0.01$ ) (Figure $4 \mathrm{~B}$ ).

IHC analysis of the mammary tumors (Figure $4 \mathrm{C}$ ) confirmed that AM9D treatment successfully downregulated MMP-9 protein expression. As shown in Figure 4C (b), AM9D treatment reduced mean MMP-9 expression by $66 \pm 11 \%$ as compared to the control DNAzyme treatment (Figure $4 \mathrm{C}$, a). This was further confirmed by the observation that the $M m p 9$ mRNA levels were $77 \%$ lower in AM9D-treated tumors compared with those tumors treated with control DNAzyme (Figure 4D). Taken together, these data show that AM9D efficiently decreases MMP-9 expression in tumors, resulting in the observed anti-tumor effects.

\section{AM9D treatment suppresses angiogenesis and stimulates apoptosis in mammary tumors}

MMP-9 has been shown to play a role in tumor progression through increase of bioavailability of VEGF and other factors that promote angiogenesis [43]. To determine the mechanism of tumor volume reduction by AM9D, the tumor slices were stained for CD-31 and for activated caspase- 3 to assess the effect of AM9D on angiogenesis and apoptosis, respectively. As shown in Figure 5A and 5B, AM9D treatment significantly reduced the number of blood vessels in the tumor as demonstrated by the lack of robust CD-31 immunostaining in the AM9D-treated group (Figure 5A, c) versus untreated (Figure 5A, a) or the control DNAzyme-treated (Figure $5 \mathrm{~A}, \mathrm{~b})$ groups. Moreover, our data also indicate that AM9D potently induces apoptosis in the tumors, as only AM9D-treated tumors contained a large number of caspase-3-positive cells, as shown in Figure 5B (b). Quantitative analysis (Figure $5 \mathrm{C}$ ) indicated that the number of CD31-positive cells was reduced 5-fold and that the intensity of the apoptotic cells increased 83-fold in tumors treated with AM9D compared to controls, respectively. These data suggest that the simultaneous 


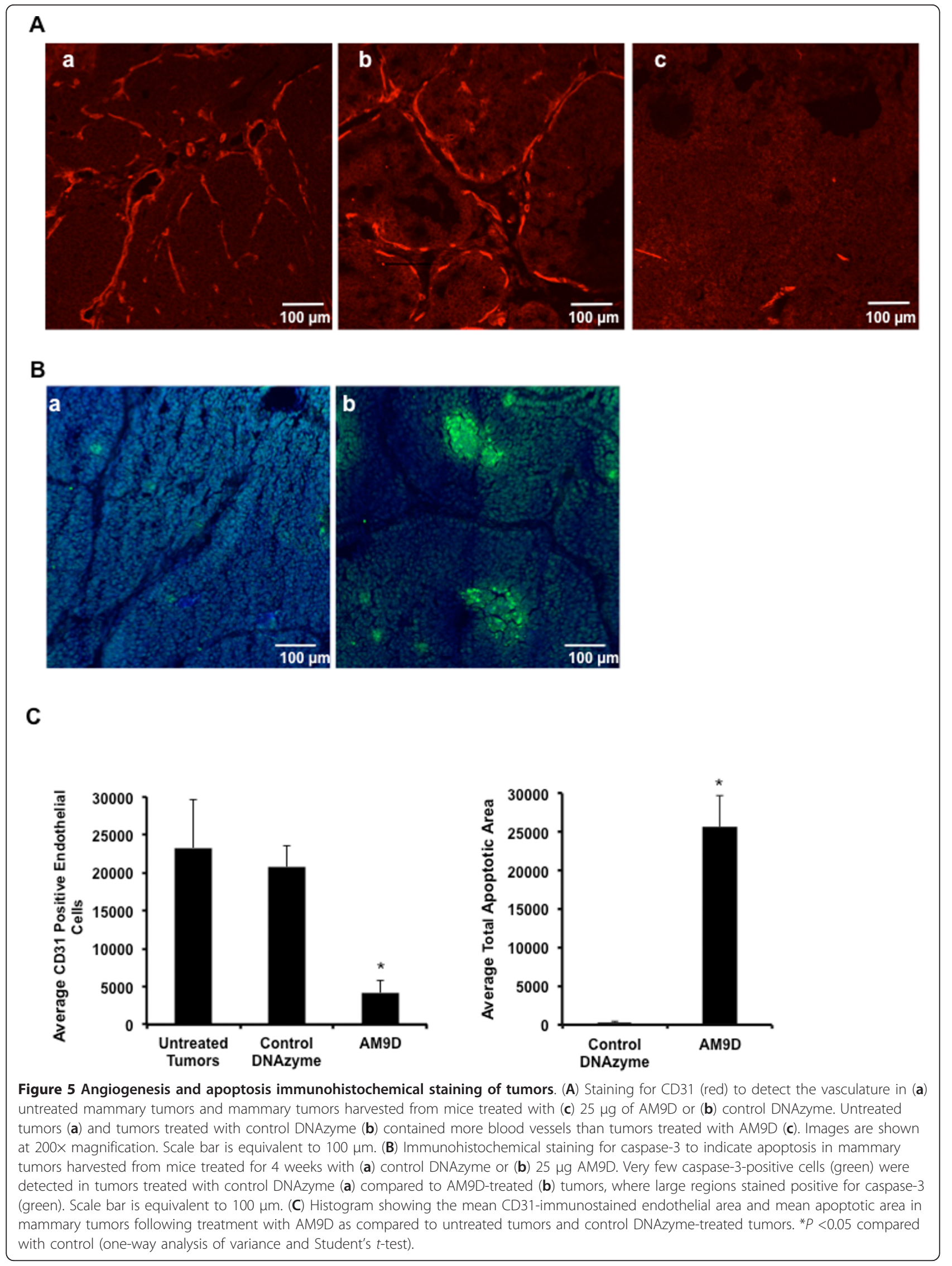


anti-angiogenic and pro-apoptotic effect of AM9D delays tumor growth over time, and decreases tumor volume at our study endpoint.

\section{Discussion}

In this study, we showed for the first time, that the downregulation of MMP-9 in mammary tumors by a novel anti-MMP-9 DNAzyme molecule results in a significant reduction in final tumor volume in the MMTV-PyMT transgenic mouse model of breast cancer. Downregulation of MMP-9 by AM9D was accompanied by a decrease in MMP-9 expression, decreased angiogenesis and increased apoptosis. Moreover, these effects were accomplished by intratumoral injection of naked DNAzyme without the use of any carriers. AMD9 treatment also reduced the invasive potential of cultured MDAMB-231 cells in vitro (Figure 1C). Together, these data indicate that specific inhibition of MMP-9 expression by DNAzyme has potential as a novel therapeutic modality to decrease the growth and invasion of carcinoma cells in the clinical setting.

It is known that MMP-9 plays a key role in angiogenesis by releasing VEGF [43] and that its downregulation induces apoptosis by stimulating the ERK pathway [17]. Martin et al. [44] have demonstrated that tumors developed in MMTV-PyMT MMP-9 wild-type mice are larger in size and are more highly vascular compared to those tumors that developed in MMTV-PyMT MMP-9null mice. Thus, these data suggest that AM9D treatment affects tumor growth via different pathways, as downregulation of MMP-9 by AM9D inhibited angiogenesis and induced apoptosis (Figure 5) as demonstrated by lack of CD31 staining and the enhanced presence of caspase-3 in AM9D-treated tumors.

Our results are consistent with those of Almholt et al. [40] in which the broad-spectrum MMP inhibitor, Galardin/GM6001, significantly reduced primary mammary tumor growth and lung metastasis in the MMTVPyMT model. However, contrary to broad-spectrum MMP inhibitors, including GM6001, AM9D treatment specifically downregulates MMP-9 without affecting the expression of other members of the MMP family. As demonstrated by the extent of cytoxicity of broad-spectrum MMP inhibitors in prior clinical trials [45-47], total inhibition of MMP is not practical. Various MMPs can exert both pro-tumorigenic and anti-tumorigenic properties [48], and some MMPs are critical for normal physiological processes, such as bone growth and remodeling, ovulation, and wound healing [49]. Further, in comparison with GM6001 [40], the intratumoral injection of AM9D not only reduced the required frequency of therapy, but was also equally efficient in reducing final tumor size. Once weekly, intratumoral injections of $25 \mu \mathrm{g}$ AM9D $(1.25 \mathrm{mg} / \mathrm{Kg})$ was sufficient to reduce the size of these spontaneously developed tumors by $50 \%$ as compared to the 51\% tumor reduction observed following daily administration of $100 \mathrm{mg} / \mathrm{Kg}$ of GM6001. Thus, the high degree of specificity of AM9D for targeting MMP-9, its in vivo stability, and the lack of any observed in vivo toxicity (Hallett M, Dalal P, Sweatman T, Pourmotabbed T: Naked Anti-Matrix Metalloproteinase-9 DNAzyme Administered Systemically Distributes to All Organs of Healthy and MMTV-PyMT Transgenic Mice and Is Safe; manuscript in review) should enhance the clinical response of solid tumors, including breast tumors, to AM9D treatment, while evading the serious side effects experienced with systemic therapy based on broad-spectrum MMP inhibitors.

The MMTV-PyMT transgenic model limited our ability to assess the efficacy of AM9D on treating spontaneous lung metastasis in vivo because not all tumors in each animal grow synchronously, and thus, not all tumors were intratumorally treated with therapy. Therefore, it was not feasible to determine the origin of metastatic cells (from treated or untreated tumors). The efficacy of AM9D in inhibiting lung metastasis is under investigation using a mouse model of metastasis.

\section{Conclusions}

Our results indicate that the downregulation of $M M P 9$ mRNA and protein expression with naked anti-MMP-9 DNAzyme is sufficient to reduce mammary tumor burden. We also describe that tumor size reduction is a result of decreased MMP-9 expression, decreased angiogenesis, and increased apoptotic cells in tumors treated with AM9D. These findings suggest specific targeting and downregulation of MMP-9 by AM9D could prove useful as a therapy against breast carcinoma tumor growth and invasion.

\section{Abbreviations \\ ANOVA: analysis of variance; a-SMA: alpha smooth muscle actin; AM9D: anti- MMP-9 DNAzyme; bp: base pairs: DAPI: 4',6-diamidino-2-phenylindole; DMEM: Dulbecco's modified Eagles medium; DNAzyme: catalytic oligodeoxynucleotide; EA: endothelial area; ECM: extracellular matrix; Egr-1: early growth response factor-1; ERK: extracellular signal-regulated kinase; FBS: fetal bovine serum; FVB: Friend virus B-type; IHC: immunohistochemistry; MMP: matrix metalloproteinase: MMTV-PyMT: mouse mammary tumor virus- polyoma virus middle T; NF-kB: nuclear factor-kB; OCT: optimal cutting temperature; PBS: phosphate-buffered saline; RT-PCR: reverse transcription- polymerase chain reaction; SE: standard error; VEGFR2: vascular endothelial growth factor receptor 2}

\section{Authors' contributions}

$\mathrm{MH}$ performed in vivo and in vitro research, participated in the design and coordination of the study, analysis and interpretation of all results, and drafting of the manuscript. BT performed in vitro research and immunohistochemistry. $\mathrm{HH}$ participated in the design, execution, acquisition, analysis and interpretation of in vitro research. LS provided training for the animal model and wrote the paper. All authors have read and approved the manuscript for publication. TS provided MMTV-PyMT transgenic mice, financial support, and wrote the paper. TP designed research, performed in vivo and in vitro research, analyzed and interpreted all the data, wrote the paper, and provided financial support. 


\section{Competing interests}

Tayebeh Pourmotabbed has applied for a patent entitled, Inhibition of tumour growth and invasion by anti-matrix metalloproteinase DNAzyme, US Divisional Patent Application Serial number 12/390,628. We have no other competing interests to declare.

\section{Acknowledgements}

We thank Dr. Kent Hunter for generously providing the MMTV-PyMT+ mice that had been previously backcrossed to the FVB/Nj strain background. We also would like to thank Drs. Yi Lu, RK Rao, Trevor Sweatman, and David Armbruster for their constructive criticism and input. Finally, we would like to thank Pooja Dalal for her assistance. This work was partially supported by USPHS GRANT CA-107183 (TP) and USPHS F31CA144572 (MH).

\section{Author details}

'Department of Microbiology, Immunology and Biochemistry, University of Tennessee Health Science Center, 858 Madison Ave., Memphis, TN 38163, USA. ${ }^{2}$ Center for Cancer Research, Department of Pathology, University of Tennessee Health Science Center, 19 S. Manassas St., Memphis TN 38163, USA.

Received: 22 May 2012 Revised: 12 January 2013

Accepted: 8 February 2013 Published: 13 February 2013

\section{References}

1. American Cancer Society: Breast Cancer Facts and Figures 2007-2008. American Cancer Society, Inc Atlanta, GA; 2009 [http://www.cancer.org].

2. Weigelt B, Peterse $\mathrm{JL}$, van 't Veer LJ: Breast cancer metastasis: markers and models. Nat Rev Cancer 2005, 5:591-602.

3. Phadke PA, Vaidya KS, Nash KT, Hurst DR, Welch DR: BRMS1 suppresses breast cancer experimental metastasis to multiple organs by inhibiting several steps of the metastatic process. Am J Pathol 2008, 172:809-817.

4. Sethi N, Kang Y: Unravelling the complexity of metastasis - molecular understanding and targeted therapies. Nat Rev Cancer 2011, 11:735-748.

5. Chambers AF, Groom AC, MacDonald IC: Dissemination and growth of cancer cells in metastatic sites. Nat Rev Cancer 2002, 2:563-572.

6. Gavrilovic J, Moens G, Thiery JP, Jouanneau J: Expression of transfected transforming growth factor alpha induces a motile fibroblast-like phenotype with extracellular matrix-degrading potential in a rat bladder carcinoma cell line. Cell Regul 1990, 1:1003-1014.

7. Lochter A, Galosy S, Muschler J, Freedman N, Werb Z, Bissell MJ: Matrix metalloproteinase stromelysin-1 triggers a cascade of molecular alterations that leads to stable epithelial-to-mesenchymal conversion and a premalignant phenotype in mammary epithelial cells. J Cell Biol 1997, 139:1861-1872.

8. Noel A, Jost M, Maquoi E: Matrix metalloproteinases at cancer tumor-host interface. Semin Cell Dev Biol 2008, 19:52-60.

9. Stuelten $\mathrm{CH}$, DaCosta Byfield $\mathrm{S}$, Arany PR, Karpova TS, Stetler-Stevenson WG, Roberts $A B$ : Breast cancer cells induce stromal fibroblasts to express MMP-9 via secretion of TNF-alpha and TGF-beta. J Cell Sci 2005, 118:2143-2153.

10. Egeblad $M$, Werb Z: New functions for the matrix metalloproteinases in cancer progression. Nat Rev Cancer 2002, 2:161-174.

11. Watanabe H, Nakanishi I, Yamashita K, Hayakawa T, Okada Y: Matrix metalloproteinase-9 (92 kDa gelatinase/type IV collagenase) from U937 monoblastoid cells: correlation with cellular invasion. J Cell Sci 1993, 104(Pt 4):991-999.

12. Cha HJ, Bae SK, Lee HY, Lee OH, Sato H, Seiki M, Park BC, Kim KW: Antiinvasive activity of ursolic acid correlates with the reduced expression of matrix metalloproteinase-9 (MMP-9) in HT1080 human fibrosarcoma cells. Cancer Res 1996, 56:2281-2284.

13. Fenhalls G, Geyp M, Dent DM, Parker Ml: Breast tumour cell-induced down-regulation of type I collagen mRNA in fibroblasts. Br J Cancer 1999, 81:1142-1149.

14. Coussens LM, Werb Z: Matrix metalloproteinases and the development of cancer. Chem Biol 1996, 3:895-904.

15. Rao JS: Molecular mechanisms of glioma invasiveness: the role of proteases. Nat Rev Cancer 2003, 3:489-501.

16. Hojilla CV, Mohammed FF, Khokha R: Matrix metalloproteinases and their tissue inhibitors direct cell fate during cancer development. Br J Cancer 2003, 89:1817-1821.
17. Bhoopathi P, Chetty C, Kunigal S, Vanamala SK, Rao JS, Lakka SS: Blockade of tumor growth due to matrix metalloproteinase- 9 inhibition is mediated by sequential activation of beta1-integrin, ERK, and NFkappaB. J Biol Chem 2008, 283:1545-1552.

18. Matrisian LM, Bowden GT, Krieg P, Furstenberger G, Briand JP, Leroy P, Breathnach R: The mRNA coding for the secreted protease transin is expressed more abundantly in malignant than in benign tumors. Proc Natl Acad Sci USA 1986, 83:9413-9417.

19. Liotta LA, Rao CN, Wewer UM: Biochemical interactions of tumor cells with the basement membrane. Annu Rev Biochem 1986, 55:1037-1057.

20. Chintala SK, Tonn JC, Rao JS: Matrix metalloproteinases and their biological function in human gliomas. Int J Dev Neurosci 1999, 17:495-502.

21. Morini M, Mottolese M, Ferrari N, Ghiorzo F, Buglioni S, Mortarini R, Noonan DM, Natali PG, Albini A: The alpha 3 beta 1 integrin is associated with mammary carcinoma cell metastasis, invasion, and gelatinase $B$ (MMP-9) activity. Int I Cancer 2000, 87:336-342.

22. Bernhard EJ, Gruber SB, Muschel RJ: Direct evidence linking expression of matrix metalloproteinase 9 (92-kDa gelatinase/collagenase) to the metastatic phenotype in transformed rat embryo cells. Proc Natl Acad Sci USA 1994, 91:4293-4297.

23. Santoro SW, Joyce GF: Mechanism and utility of an RNA-cleaving DNA enzyme. Biochemistry 1998, 37:13330-13342.

24. Santoro SW, Joyce GF: A general purpose RNA-cleaving DNA enzyme. Proc Natl Acad Sci USA 1997, 94:4262-4266.

25. Dass CR, Choong PF, Khachigian LM: DNAzyme technology and cancer therapy: cleave and let die. Mol Cancer Ther 2008, 7:243-251.

26. Lowe HC, Fahmy RG, Kavurma MM, Baker A, Chesterman CN, Khachigian LM: Catalytic oligodeoxynucleotides define a key regulatory role for early growth response factor- 1 in the porcine model of coronary in-stent restenosis. Circ Res 2001, 89:670-677.

27. Khachigian LM, Fahmy RG, Zhang G, Bobryshev YV, Kaniaros A: c-Jun regulates vascular smooth muscle cell growth and neointima formation after arterial injury. Inhibition by a novel DNA enzyme targeting c-Jun. J Biol Chem 2002, 277:22985-22991.

28. Zhang L, Gasper WJ, Stass SA, loffe OB, Davis MA, Mixson AJ: Angiogenic inhibition mediated by a DNAzyme that targets vascular endothelial growth factor receptor 2. Cancer Res 2002, 62:5463-5469.

29. Sun LQ, Cairns MJ, Saravolac EG, Baker A, Gerlach WL: Catalytic nucleic acids: from lab to applications. Pharmacol Rev 2000, 52:325-347.

30. Hegedüs L, Cho H, Xie X, Eliceiri GL: Additional MDA-MB-231 breast cancer cell matrix metalloproteinases promote invasiveness. J Cell Physiol 2008, 216:480-485.

31. Bu CH, Pourmotabbed T: Mechanism of activation of human neutrophil gelatinase B: Discriminating between the role of $\mathrm{Ca}$ in activation and catalytsis. J Biol Chem 1995, 270: 18563-18569.

32. Chantrain CF, DeClerck YA, Groshen S, McNamara G: Computerized quantification of tissue vascularization using high-resolution slide scanning of whole tumor sections. J Histochem Cytochem 2003, 51:151-158.

33. Yodkeeree S, Ampasavate C, Sung B, Aggarwal BB, Limtrakul P: Demethoxycurcumin suppresses migration and invasion of MDA-MB-231 human breast cancer cell line. Eur J Pharmacol 2010, 627:8-15.

34. Lin EY, Jones JG, Li P, Zhu L, Whitney KD, Muller WJ, Pollard JW Progression to malignancy in the polyoma middle T oncoprotein mouse breast cancer model provides a reliable model for human diseases. Am J Pathol 2003, 163:2113-2126.

35. Fantozzi A, Christofori G: Mouse models of breast cancer metastasis. Breast Cancer Res 2006, 8:212

36. Herschkowitz JI, Simin K, Weigman VJ, Mikaelian I, Usary J, Hu Z, Rasmussen KE, Jones LP, Assefnia S, Chandrasekharan S, Backlund MG, Yin Y, Khramtsov IA, Bastein R, Quackenbush J, Glazer RI, Brown PH, Green JE, Kopelovich L, Furth PA, Palazzo JP, Olopade OI, Bernard PS, Churchill GA, Dyke TV, Perou CM: Identification of conserved gene expression features between murine mammary carcinoma models and human breast tumors. Genome Biol 2007, 8:R76.

37. Pedersen TX, Pennington CJ, Almholt K, Christensen IJ, Nielsen BS, Edwards DR, Romer J, Dano K, Johnsen M: Extracellular protease mRNAs are predominantly expressed in the stromal areas of microdissected mouse breast carcinomas. Carcinogenesis 2005, 26:1233-1240.

38. Szabova L, Yamada SS, Birkedal-Hansen H, Holmbeck K: Expression pattern of four membrane-type matrix metalloproteinases in the normal and diseased mouse mammary gland. J Cell Physiol 2005, 205:123-132. 
39. Almholt K, Green KA, Juncker-Jensen A, Nielsen BS, Lund LR, Romer J: Extracellular proteolysis in transgenic mouse models of breast cancer. J Mammary Gland Biol Neoplasia 2007, 12:83-97.

40. Almholt K, Juncker-Jensen A, Laerum OD, Dano K, Johnsen M, Lund LR, Romer J: Metastasis is strongly reduced by the matrix metalloproteinase inhibitor Galardin in the MMTV-PymT transgenic breast cancer model. Mol Cancer Ther 2008, 7:2758-2767.

41. Lin EY, Nguyen AV, Russell RG, Pollard JW: Colony-stimulating factor 1 promotes progression of mammary tumors to malignancy. J Exp Med 2001, 193:727-740.

42. Lin EY, Gouon-Evans V, Nguyen AV, Pollard JW: The macrophage growth factor CSF-1 in mammary gland development and tumor progression. $J$ Mammary Gland Biol Neoplasia 2002, 7:147-162.

43. Bergers G, Brekken R, McMahon G, Vu TH, Itoh T, Tamaki K, Tanzawa K, Thorpe P, Itohara S, Werb Z, Hanahan D: Matrix metalloproteinase-9 triggers the angiogenic switch during carcinogenesis. Nat Cell Biol 2000 2:737-744

44. Martin MD, Carter KJ, Jean-Philippe SR, Chang M, Mobashery S, Thiolloy S, Lynch CC, Matrisian LM, Fingleton B: Effect of ablation or inhibition of stromal matrix metalloproteinase- 9 on lung metastasis in a breast cancer model is dependent on genetic background. Cancer Res 2008, 68:6251-6259.

45. Smyth E: The trouble with inhibitors. Signalling Scissors: New Perspectives on Proteases, Horizon Symposia, Brescia, Italy, Nature Publishing Group 2003, 1-4[http://www.nature.com/horizon/proteases/background/pdf/inhibitors. pdf].

46. Wojtowicz-Praga SM, Dickson RB, Hawkins MJ: Matrix metalloproteinase inhibitors. Invest New Drugs 1997, 15:61-75.

47. Rasmussen HS, McCann PP: Matrix metalloproteinase inhibition as a novel anticancer strategy: a review with special focus on batimastat and marimastat. Pharmacol Ther 1997, 75:69-75.

48. Martin MD, Matrisian LM: The other side of MMPs: protective roles in tumor progression. Cancer Metastasis Rev 2007, 26:717-724.

49. Coussens LM, Fingleton B, Matrisian LM: Matrix metalloproteinase inhibitors and cancer: trials and tribulations. Science 2002, 295:2387-2392.

doi:10.1186/bcr3385

Cite this article as: Hallett et al: Anti-matrix metalloproteinase- 9 DNAzyme decreases tumor growth in the MMTV-PyMT mouse model of breast cancer. Breast Cancer Research 2013 15:R12.

\section{Submit your next manuscript to BioMed Central and take full advantage of:}

- Convenient online submission

- Thorough peer review

- No space constraints or color figure charges

- Immediate publication on acceptance

- Inclusion in PubMed, CAS, Scopus and Google Scholar

- Research which is freely available for redistribution

Submit your manuscript at www biomedcentral.com/submit
Biomed Central 